ISSN e- 2594-1100

en-claves del pensamiento / núm. 31 / enero-junio / 2022 / e498

https://doi.org/10.46530/ecdp.v0i31.498 DOSSIER (enn-claves

\title{
VIOLENCIA VIRTUAL CONTRA EL COLECTIVO LGBTIQ+: UNA REVISIÓN SISTEMÁTICA
}

\author{
Virtual Violence Against LGBTIQ+ People: \\ A Systematic Review
}

\author{
Raquel Lozano-Blasco, Universidad de Zaragoza \\ Correo electrónico: rlozano@unizar.es
}

Alberto Soto-Sánchez, Universidad de Valladolid

Correo electrónico: alberto.soto@uva.es

Recibido: $15 / 05 / 2021$

Aceptado: $15 / 11 / 2021$

Publicado: 07/01/2022

\begin{abstract}
Resumen. El presente trabajo muestra la vulnerabilidad del colectivo LGBTIQ+ a la hora de recibir agresiones a través de las nuevas tecnologías, en lo que se conoce como cyberbullyng, ciberacoso o violencia virtual, por no ajustarse a un perfil heteronormativo, observándose este tipo de agresiones también en instituciones educativas que muestran acciones y políticas basadas en estereotipos no inclusivos. La revisión se ha llevado a cabo mediante una búsqueda en diversas bases de datos científicas y un análisis siguiendo el manual de revisiones sistemáticas de Cochrane, en Higgins y Green y PRISMA. Se concluye que cyberbulliyng LGBTIQ+ fóbico muestra una prevalencia creciente en los últimos años, con importantes consecuencias socioemocionales y conductuales para el colectivo vulnerable, que hacen necesaria una intervención en materia de sensibilización y concienciación dentro los centros educativos.
\end{abstract}

Palabras clave: ciberacoso, violencia, LGBTIQ+ adolescencia, vulnerabilidad.

\begin{abstract}
This work shows the vulnerability of the LGBTIQ + community to receive attacks through new technologies. This is known as cyberbullying or virtual violence, and it occurs because this social group does not present a heteronormative profile. In addition, this type of aggression is also observed in educational institutions which show actions and policies based on non-inclusive stereotypes. For this review, a scientific database search and analysis has been carried out following the Cochrane systematic reviews manual, in Higgins and Green and PRISMA. It is concluded that LGBTIQ + phobic cyberbullying shows an increasing prevalence in recent years, with important socio-emotional and behavioral consequences. An intervention is necessary in terms of sensitization and awareness within educational centers.
\end{abstract}

Keywords: cyberbullying, violence, LGBTIQ+ adolescence, vulnerability.

Cómo citar: Lozano-Blasco, R., y Soto-Sánchez, A. (2022). Violencia virtual contra el colectivo LGBTIQ+: una revisión sistemática. EN-CLAVES del pensamiento, 0(31), e498. doi: https://doi.org/10.46530/ecdp.v0i31.498 


\section{Introducción}

El cyberbulliyng LGBTIQ+ fóbico hace referencia a cualquier tipo de agresión destinada a personas cuya orientación sexual real o percibida, la de un familiar o amistad de la víctima, ${ }^{1}$ no se ajuste a los patrones heteronormativo, ${ }^{2}$ mediante el uso de las nuevas tecnologías de comunicación. De este modo, no solo afecta al colectivo de personas homosexuales sino que afecta a toda persona que no cumpla el estereotipo de masculinidad/feminidad tradicional estipulados en los patrones heteronormativos. ${ }^{3}$ Aunque no existe plena conformidad respecto a la definición, ${ }^{4}$ la literatura presente define este tipo de violencia desde una visión de multidimensionalidad, en la cual se manifiesta hostigamiento contra la diversidad sexual y de género en general. ${ }^{5}$ De este modo, la víctima es excluida, aislada y/o amenazada, recibiendo agresiones de manera constante, es decir, los efectos negativos se experimentan a nivel emocional (depresión, baja autoestima), conductual (aislamiento) y en rendimiento académico, ${ }^{6}$ disminuyendo su autoestima e incrementando el nivel de depresión, estrés e idealización suicida. ${ }^{7}$

\footnotetext{
${ }^{1}$ Santiago Agustín, Diversidad sexual en las aulas. Evitar el bullying o acoso. (Badajoz: Fundación Trián, 2009).

${ }^{2}$ Paz Elipe, María de la Oliva Muñoz, María y Rosario Del Rey, "Homophobic Bullying and Cyberbullying: Study of a Silenced Problem", Journal of Homosexuality, núm. 65 (2018): 672-686. https://doi.org/10.1080/00918369.2017.1333809; Enara Larraina y Maite Garaigordobil, "El bullying en el País Vasco: prevalencia y diferencias en función del sexo y la orientación-Sexual”, Clínica y Salud, núm. 31 (2020): 147-153; Delia Socarrás, Massiel Nuñez, Martín del Río Marais, Ada Rodríguez y Gisett Gutiérrez, "Estudio exploratorio retrospectivo sobre violencia homofóbica y transfóbica en la trayectoria escolar de activistas cubanos LGBT”, Revista Sexología y Sociedad, núm. 25 (2019): 72-87.

${ }^{3}$ Andreas Gegenfurtner y Markus Gebhardt, "Sexuality education including lesbian, gay, bisexual, and transgender (LGBT) issues in schools", Educational Research Review, núm. 22 (September 2017): 215-222. https://doi.org/10.1016/j.edurev.2017.10.002.

${ }^{4}$ José Ignacio Pichardo, Abrazar la diversidad: propuestas para una educación libre de acoso homofóbico y transfóbico (Madrid: Instituto de la Mujer y para la Igualdad de Oportunidades, 2015).

${ }^{5}$ Francisca Cifuentes, Cristian Carrer y Javier Pascual, "Acoso escolar por orientación sexual, identidad y expresión de género en institutos de educación secundaria catalanes", Revista Nacional e Internacional de Educación Inclusiva, núm. 13 (2020): 153-174 Carmen Ruiz, "Estrategias para educar en y para la igualdad: coeducar en los centros", ATLÁNTICAS - Revista Internacional de Estudios Feministas, núm. 2 (2017): 166-191.

${ }^{6}$ Roberto Abreu y Maureen Kenny, "Cyberbullying and LGBTQ Youth: A Systematic Literature Review and Recommendations for Prevention and Intervention", Journal of Child and Adolescent Trauma, núm. 11 (2018): 81-97. https://doi.org/10.1007/s40653-017-0175-7.

${ }^{7}$ Alida Bouris, Bethany Everett, Ryan Heath, Caitlin Elsaesser y Torsten Neilands, "Effects of Victimization and Violence on Suicidal Ideation and Behaviors among Sexual Minority and Heterosexual Adolescents". LGBT Health, núm. 3 (2016): 153-161. https://doi.org/10.1089/lgbt.2015.0037. Chad Burton, Michael Marshal, Deena Chisolm, Gina Sucato y Mark Friedman Mark, "Sexual Minority-related Victimization as a Mediator of Mental Health Disparities in Sexual Minority Youth: A Longitudinal Analysis", Journal of Youth and Adolescence, núm. 42 (2013): 394-402; Kate Collier, Gabriel van Beusekom, Henry Bos y Theo
} 
Por otra parte, no se debe obviar cómo el cyberbullying LGBTIQ+ fóbico también se puede desarrollar en las instituciones educativas mediante acciones y políticas basadas en los estereotipos y sin un planteamiento inclusivo. En este sentido, Pichardo ${ }^{8}$ recuerda cómo la homofobia no opera en lo irracional o en el subconsciente, sino que es un prejuicio construido socialmente en el que se aprende a rechazar la diversidad. En este sentido, es necesario referenciar como Cifuentes ${ }^{9}$ explica los tres conceptos esenciales en la configuración de comportamientos y actitudes LGBTIQ+ Fóbicos: a) estereotipos, siendo estos entendidos como simplificaciones y asociaciones de ideas, ${ }^{10}$ b) actitudes, siendo estas la organización de creencias y cogniciones, ${ }^{11}$ y c) prejuicios, siendo una predisposición negativa hacia un colectivo. ${ }^{12}$ Por otra parte, Dunn et al. ${ }^{13}$ exponen que la misoginia o la aversión a la feminidad es un elemento de riesgo en la aparición del acoso, pudiendo ser esta la causa de que las mujeres y hombres de minorías sexuales sufran en mayor medida acoso. Si bien otras investigaciones como Edwards et al. ${ }^{14}$ mostraron que la orientación sexual de las mujeres no modera el ciberacoso que sufren, estas eran en mayor medida víctimas en comparación con sus homólogos. Por otra parte, es necesario exponer cómo los hombres homosexuales tenían mayor probabilidad de sentirse amenazados que sus homólogos ante una situación de ciberacoso. ${ }^{15}$

Sandfort, "Sexual Orientation and Gender Identity/Expression Related Peer Victimization in Adolescence: A Systematic Review of Associated Psychosocial and Health Outcomes", Journal of Sex Research, núm. 50 (2013): 299-317.

${ }^{8}$ Pichardo, Abrazar la diversidad, 15.

${ }^{9}$ Cifuentes, Carrer y Pascual, "Acoso escolar", 154.

${ }^{10}$ Margarita Del Olmo, "Prejuicios y estereotipos: un replanteamiento de su uso y utilidad como mecanismos sociales", Revista de Educación XXI, núm. 7 (2005): 13-24.

${ }^{11}$ Carlos Parales-Quenza y Milcíades Vizcaíno-Gutiérrez, "Las relaciones entre actitudes y representaciones sociales: elementos para una integración conceptual". Revista Latinoamericana de Psicología, núm. 39 (2007): 351-361; Arantxa Vizcaíno Verdú, Paloma Contreras Pulido y María Dolores Guzmán Franco, “'La Bella y la Bestia' y el movimiento LGBTIQ+ en Disney: empoderamiento y activismo en YouTube", Área Abierta, núm. 20 (2020): 75-93.

${ }^{12}$ Del Olmo, "Prejuicios y estereotipos", 15.

${ }^{13}$ Hailee Dunn, Melissa Clark y Deborah Pearlman, "The Relationship Between Sexual History, Bullying Victimization, and Poor Mental Health Outcomes Among Heterosexual and Sexual Minority High School Students: A Feminist Perspective”. Journal of Interpersonal Violence, núm. 32 (2017): 3497-3519.

${ }^{14}$ Katie Edwards, Kateryna Sylaska, Johanna Barry, Mary Moynihan, Victoria Banyard, Ellen Cohn, Wendy Walsh y Sally Ward, "Physical Dating Violence, Sexual Violence, and Unwanted Pursuit Victimization: A Comparison of Incidence Rates among Sexual-minority and Heterosexual College Students", Journal of Interpersonal Violence, núm. 30 (2015): 580-600. https://doi.org/10.1177/0886260514535260.

${ }^{15}$ Robert Tait, "Alcohol-related victimisation: Differences between Sexual Minorities and Heterosexuals in an Australian National Sample", Drug and Alcohol Review, núm. 34 (2015): 366-374. 
En cuanto al perfil del agresor, es necesario exponer que no existe un perfil unívoco, pero sí existe conformidad en una serie de aspectos, como los elevados prejuicios y disposición poco tolerante ante la diversidad afectivo-sexual, ${ }^{16}$ dificultades en inteligencia emocional y también en empatía, ${ }^{17}$ falta de información sobre este colectivo $^{18}$ o bajo nivel socio-educativo, ${ }^{19}$ siendo estos no sólo elementos propios de la psicología de las diferencias individuales, sino factores culturales y sociales de la comunidad, indicando que el entorno es una variable determinante. ${ }^{20}$ Del mismo modo, el establecimiento de un clima de seguridad y tranquilidad en los centros educativos permite a los estudiantes que forman parte de este colectivo desarrollarse con plena libertad y tranquilidad, mejorando de manera significativa su calidad de vida. Esta situación ocurre prioritariamente en Educación Secundaria, durante el periodo de la adolescencia, ${ }^{21}$ si bien se mantiene hasta la educación universitaria. ${ }^{22} \mathrm{El}$ efecto del estrés social, desde la teoría de Meyer, ${ }^{23}$ que sufre este colectivo disminuye su calidad de vida, ${ }^{24}$ aumentando la prevalencia a sufrir depresión, estrés y baja autoestima. ${ }^{25}$

Las redes sociales no solo pueden ser origen de malas experiencias, sino que pueden generarse como un espacio de apoyo en el cual se fomentan relaciones positivas, aumentando la red de apoyo y la sensibilización de la sociedad. Estas acciones ayudarían

\footnotetext{
${ }^{16}$ Rocío Garrido y Zaira Morales, "Una aproximación a la Homofobia desde la Psicología", Propuestas de Intervención Psicología, Conocimiento y Sociedad, núm. 4 (2014): 90-115.

${ }^{17}$ Larrain y Garaigordobil, "El bullying en el País Vasco", 149.

18 Mark Hatzenbuehler, Dustin Duncan y Renee Johnson, "Neighborhood-level LGBT Hate Crimes and Bullying among Sexual Minority Youths: A Geospatial Analysis". Violence and Victims, núm. 30 (2015): 663-675. https://doi.org/10.1891/0886-6708.VV-D-13-00166.

${ }^{19}$ Gegenfurtner y Gebhardt, "Sexuality education", 217.

${ }^{20}$ Clayton Cook, Kirk Williams, Nancy Guerra, Tia Kim y Shelly Sadek, "Predictors of Bullying and Victimization in Childhood and Adolescence: A Meta-Analytic Investigation", School Psychology Quarterly, núm. 25 (2010): 65-83.

${ }^{21}$ Dorothy Espelage, Kathleen Basile y Merle Hamburger, "Bullying Perpetration and Subsequent Sexual Violence Perpetration among Middle School Students". Journal of Adolescent Health, núm. 50 (2012): 60-65.

${ }^{22}$ Susan Rankin, Genevieve Weber, Warren Blumenfeld y Somjen Frazer, State of Higher Education for Lesbian, Gay, Bisexual, and Transgender People: 2010 National College Climate Survey (Charlotte, NC: Campus Pride, 2010); Jaimi Ramsey, Lisabeth DiLalla y Megan McCrary, "Cyber Victimization and Depressive Symptoms in Sexual Minority College Students", Journal of School Violence, núm. 15 (2016): 483-502.

${ }^{23}$ Ilan Meyer, "Prejudice, social stress, and mental health in lesbian, gay, and bisexual populations: Conceptual issues and research evidence", Psychological Bulletin, núm. 129 (2003): 674-697.

${ }^{24}$ David Frost y Ilan Meyer, "Internalized Homophobia and Relationship Quality among Lesbians, Gay Men, and Bisexuals". Journal of Counseling Psychology, núm. 56 (2009): 97-109. https://doi.org/10.1037/a0012844.

${ }^{25}$ Dunn, Clark y Pearlman, "The relationship between sexual history", 3501.
} 
a reducir el estrés y la depresión experimentados por la comunidad LGBTIQ $+{ }^{26}$ La complejidad de este tipo de violencia, unida a las alarmantes tasas de prevalencia que sufre este colectivo (ver tabla 1) en comparación a sus iguales, oscilando entre un 10.5 por ciento y un 71.3 por ciento, junto a una perspectiva inclusiva desde el cual parten las acciones sociales, han provocado el crecimiento de acciones educativas en diferentes naciones con la finalidad de educar en diversidad sexual. ${ }^{27}$

Tabla 1. Prevalencia del ciberacoso en el colectivo LGBTIQ+

\begin{tabular}{|c|c|c|}
\hline Autor & Prevalencia & Nación \\
\hline Mayock et al. (2009) & $\begin{array}{l}\text { El } 40 \% \text { fue víctima de agresiones } \\
\text { verbales de carácter homofóbico. }\end{array}$ & Europeo \\
\hline $\begin{array}{l}\text { Abreu, R. L. y Kenny, } \\
\text { M. C. (2017). }\end{array}$ & $\begin{array}{l}\text { Entre } 10.5 \% \text { y } 71.3 \% \text { de víctimas del } \\
\text { colectivo LGBT. }\end{array}$ & $\begin{array}{l}\text { Internacional } \\
\text { Estudio de revisión } \\
\text { sistemática }\end{array}$ \\
\hline COGAM (2016). & $\begin{array}{l}\text { El } 70 \% \text { de las personas LGB ha sufrido } \\
\text { o sufre cyberbullying. }\end{array}$ & España \\
\hline Kosciw et al. (2016). & $\begin{array}{l}\text { El } 85.2 \% \text { estudiantes LGBTQ } \\
\text { experimentaron acoso verbal (por } \\
\text { ejemplo, insultos o amenazas). }\end{array}$ & USA \\
\hline $\begin{array}{l}\text { Hinduja } \quad \text { y } \quad \text { Patchin } \\
(2011) .\end{array}$ & $\begin{array}{l}\text { El } 72 \% \text { de los estudiantes LGBT fueron } \\
\text { víctimas frente al } 63 \% \text { de los } \\
\text { estudiantes heterosexuales. }\end{array}$ & USA \\
\hline (Guasp, 2012). & $\begin{array}{l}\text { El } 55 \% \text { de los estudiantes LGB sufren } \\
\text { acoso homófobo. }\end{array}$ & Reino Unido \\
\hline Martxueta (2014). & $\begin{array}{l}\text { El } 57.4 \% \text { de las víctimas de ciberacoso } \\
\text { pertenecían a la comunidad LGBTIQ+. }\end{array}$ & España \\
\hline
\end{tabular}

El objetivo del presente trabajo es determinar la incidencia del ciberacoso en adolescentes del colectivo LGBTIQ+ en los últimos años (2016-2020).

\section{Metodología}

La implementación del protocolo de investigación se efectuó atendiendo a las especificaciones del manual de revisiones sistemáticas de Cochrane en Higgins y Green ${ }^{28}$ y

${ }^{26}$ Begonya Enguix, "Activismo y Prácticas Digitales en la Construcción de una Esfera LGBTIQ+ en España”. Dados, núm. 59 (2016): 755-787. https://doi.org/10.1590/00115258201691.

27 James Ponzetti, Evidence-based Approaches to Sexuality Education. A Global Perspective (New York: Routledge, 2016).

${ }^{28}$ Julian Higgins y Sally Green, Cochrane Handbook for Systematic Reviews of Interventions Version 5.1.0 (London: The Cochrane Collaboration, 2011). 
PRISMA. ${ }^{29}$ Por otra parte, los criterios de inclusión y exclusión se suscribieron a lo estipulado por Botella y Sánchez ${ }^{30}$ y Moreau and Gamble: ${ }^{31}$

- Fecha de publicación: 2016-2020.

- Rigurosidad metodológica y ética: evidencia clara de seguimiento de método científico y aplicación de criterios éticos en el proceso de investigación de acuerdo con los principios de PICO. ${ }^{32}$

- Instrumentos de medida: las variables habrán sido medidas mediante pruebas psicométricas baremadas.

- Idioma: inglés o castellano.

Por lo que respecta a los criterios de exclusión se determinaron según las indicaciones de Botella y Sánchez ${ }^{33}$ y Moreau and Gamble: ${ }^{34}$

- La determinación de datos incongruentes, imprecisos o la ausencia básica de tratamiento de datos, tanto de naturaleza cualitativa como cuantitativa, así como la baremación incorrecta de los instrumentos psicométricos son factores que implican el rechazo de la investigación.

\section{Acciones booleanas empleadas en la estrategia}

La estrategia de búsqueda. Esta se realizó en tres bases de datos: Psycoinfo, Scopus y Proquest. La búsqueda se efectuó en abril de 2021. Se seleccionó la siguiente acción booleana: "cyberbullying OR cybervictims OR cyberbullies AND xenophobia" (ver figura 1). Se establecieron los siguientes filtros:

- Horquilla temporal: 2016-2021

- Scopus: "article title, abstract, keywords" "article".

${ }^{29}$ David Moher, Alessandro Liberati, Jennifer Tetzlaff, Douglas Altman y PRISMA Group. "Preferred Reporting Items for Systematic Reviews and MetaAnalyses: The PRISMA Statement", PLoS Medicine, núm. 6 (2009): e1000097. https://doi.org/10.1371/journal.pmed.1000097.

${ }^{30}$ Juan Botella y Julio Sánchez, Meta-análisis en ciencias sociales y de la salud (Madrid: Síntesis, 2015).

${ }^{31}$ David Moreau y Beau Gamble, Conducting a Meta-analysis in the Age of Open Science: Tools, Tips, and Practical Recommendations (Auckland: The University of Auckland, 2020).

${ }^{32}$ Cristina Mamédio da Costa Santos, Cibele de Mattos Pimenta y Moacyr Cuce Nobre, "Estrategia pico para la construcción de la pregunta de investigación y la búsqueda de evidencias", Revista Latino-americana de Enfermagem, núm. 15 (2007): 2-4.

${ }^{33}$ Botella y Sánchez, Meta-análisis en ciencias sociales, 21.

${ }^{34}$ Moreau y Gamble, Conducting a meta-analysis, 15. 
- Psycinfo: "keywords", "texto completo" "article".

- Proquest: "title, abstract o key words", "texto completo", "article".

Figura 1. Gráfico de flujo

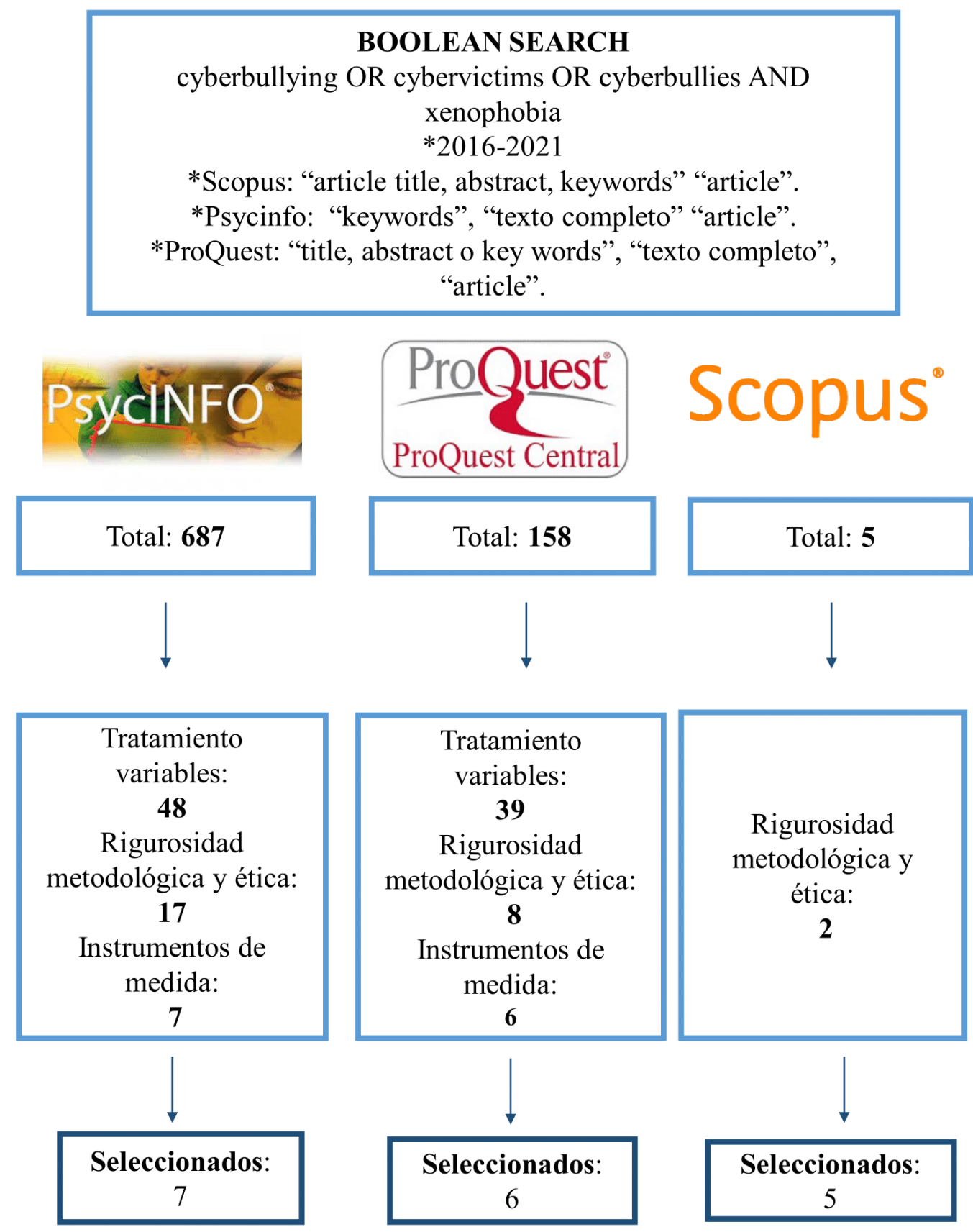

Se codificaron de manera manual y sistemática cada una de las investigaciones mediante la lectura del abstract, título y palabras clave. Este proceso permite determinar qué investigaciones tratan la temática. Igualmente, es necesario indicar que en este proceso la 
mayor parte de los resultados fueron excluidos, pues en su mayoría no trataban ambas variables. En segundo lugar, se aplicaron los criterios de inclusión y exclusión.

\section{Resultados}

\section{Descripción demográfica}

Recientemente, entre los años 2016 y 2021, se han desarrollado interesantes investigaciones que persiguen la finalidad de estudiar en detenimiento la relación entre pertenecer a minorías sexuales y el ciberacoso. En total, la revisión sistemática se compone de 14 estudios con 15 muestras (una de las investigaciones es longitudinal) que suman un total de 38,164 adolescentes y jóvenes con una edad media de 17.72 años. En lo referente al tamaño de las muestras (véase tabla 2), la más pequeña se compone de 125 y la mayor de 19,082 participantes. Además, la revisión aúna investigaciones europeas (Francia, Italia, Bélgica, España), norteamericanas (EE. UU. y Canadá) y asiáticas (Taiwan), tal y como se puede observar en la tabla 3. En cuanto a la antropología, es necesario referenciar que la mayor parte de la muestra concierne a países europeos, siendo esto indicativo de la necesidad de estudio y concienciación en otras zonas geográficas.

Tabla 2. Distribución de población

\begin{tabular}{|l|l|l|l|l|l|l|l|l|l|}
\hline Nación & Bélgica & Canadá & España & Francia & Israel & Italia & Suecia & Taiwan & EE.UU. \\
\hline Muestra & 2074 & 1748 & 4065 & 1889 & 347 & 3470 & 4989 & 500 & 19082 \\
\hline
\end{tabular}

Tabla 3. Datos sociodemográficos de la muestra

\begin{tabular}{|l|l|l|l|}
\hline Referencia & $\begin{array}{l}\text { Tamaño } \\
\text { muestra }\end{array}$ & Edad & Nacionalidad \\
\hline (Sterzing et al., 2016) & 125 & $15-19$ & EE. UU. \\
\hline (Camodeca et al., 2019) & 197 & 16.32 & Italia \\
\hline (Dermody et al., 2016) & 290 & 17.08 & EE. UU. \\
\hline (Zerach, 2016) & 347 & -- & Israel \\
\hline (Otis et al., 2016) & 400 & 32.8 & EE. UU. \\
\hline (Wang et al., 2018) & 500 & $20-25$ & Taiwán \\
\hline (continúa) & & & \\
\hline
\end{tabular}




\begin{tabular}{|l|l|l|l|}
\hline (Elipe et al., 2018) & 533 & 14.9 & España \\
\hline (Desmet et al., 2021) & 1,037 & 15.2 & Bélgica \\
\hline (Desmet et al., 2018) & 1,037 & $12-18$ & Bélgica \\
\hline (Garaigordobil y Larrain, 2020) & 1,784 & $13-17$ & España \\
\hline (Blaya y Audrin, 2019) & 1,889 & 14.63 & Francia \\
\hline (Tintori et al., 2021) & 3,273 & 16 & Italia \\
\hline (Donahue et al., 2017) & 4,989 & 18 & Suecia \\
\hline Larrain y Garaigorgobil (2020) & 1,748 & $14-18$ & España \\
\hline
\end{tabular}

\section{Discusión y conclusiones}

La revisión sobre el cyberbulliyng LGBTIQ+ fóbico muestra una prevalencia creciente. La evidencia científica nos dice que, en los últimos años, entre el 10.5 por ciento y el 71.30 por ciento del colectivo ha sufrido ciberviolencia. ${ }^{35}$ Estas cifras son semejantes al estudio de COGAM,${ }^{36}$ quien expone que el 70 por ciento de las personas de este colectivo ha sufrido o está sufriendo ciberacoso en España. Aunque no existe un modo único de ciberagresión, ${ }^{37}$ la intensidad y cronicidad de estos episodios de humillación, aislamiento y hostigamiento provocan importantes consecuencias emocionales en las víctimas. ${ }^{38}$ De este modo, pertenecer al colectivo LGBTIQ+ implica mayor probabilidad de padecer ciberacoso. ${ }^{39}$ Además, la evidencia científica expone que las mujeres de este colectivo se encuentran afectadas por este problema en mayor medida que sus compañeros de sexo masculino. ${ }^{40}$

\footnotetext{
${ }^{35}$ Abreu y Kenny, "Cyberbullying and LGBTQ Youth”, 83

36 Eduardo Benítez, Ciberbullying LGBT-fóbico. Nuevas formas de intolerancia. (Madrid: Grupo de Educación de COGAM, 2016).

${ }^{37}$ Carmen Ruiz, "Estrategias para educar en y para la igualdad: coeducar en los centros". ATLÁNTICAS Revista Internacional de Estudios Feministas, núm. 2 (2017): 166-191.

${ }^{38}$ Larrain y Garaigordobil, "El bullyng en el País Vasco", 151.

${ }^{39}$ Marina Camodeca, Roberto Baiocco y Ortensia Posa, "Homophobic Bullying and Victimization among Adolescents: The Role of Prejudice, Moral Disengagement, and Sexual Orientation”, European Journal of Developmental Psychology, núm. 16 (2019): 503-521. https://doi.org/10.1080/17405629.2018.1466699; Maite Garaigordobil y Enara Larrain, "Bullying and cyberbullying in LGBT adolescents: Prevalence and effects on mental health", Comunicar, núm. 28 (2020): 77-87. https://doi.org/10.3916/C62-2020-07.

${ }^{40}$ Melanie Otis, Carrie Oser y Michele Staton-Tindall, "Violent Victimization and Substance Dependency: Comparing Rural Incarcerated Heterosexual and Sexual Minority Women”, Journal of Social Work Practice in the Addictions, núm. 16 (2016): 176-201; Lihien-Chuan Wang, Mu-Hong Chen, Nai-Ying Ko, Yu-Ping Chang, Huang-Chi Lin y Cheng-Fan Yen, "Effects of Traditional and Cyber Homophobic Bullying in Childhood on Depression, Anxiety, and Physical Pain in Emerging Adulthood and the Moderating Effects of
} 
En lo que respecta a las consecuencias emocionales, encontramos unanimidad en los resultados de la revisión sistemática. La tasa de angustia, ansiedad, idealización suicida, depresión, baja autoestima, aislamientos y bajo rendimiento académico entrañan un perfil complejo, en el que la víctima se siente desbordada por la situación e incluso presenta un mayor consumo de estupefacientes. ${ }^{41}$ En este sentido, la orientación sexual sí que media en la intensidad de las consecuencias socio-emocionales, pues tanto las cibervíctimas como los ciberagresores con una orientación no-heterosexual sufren mayores tasas de depresión, sintomatología patológica a excepción de ansiedad social. ${ }^{42}$ Además, es necesario referenciar que las consecuencias psicológicas del ciberacoso también pueden llegar a mantenerse en la edad adulta. ${ }^{43}$

En lo relativo a los acosadores, no encontramos un perfil definido. De inicio, algunas investigaciones lo asocian al sexo masculino y a una orientación sexual heterosexual, ${ }^{44}$ mientras que otras exponen que no hay diferencias significativas en la orientación sexual y el sexo de los agresores. ${ }^{45}$ Sin embargo, sí existe consenso en cuanto al perfil psicológico. De inicio, al igual que las víctimas, los agresores experimentan en mayor medida depresión,

Social Support among Gay and Bisexual Men in Taiwan”, Neuropsychiatric Disease and Treatment, núm. 14 (2018): 1309-1317.

${ }^{41}$ Paul Sterzing, Jun Sung Hong, Rachel Gartner y Wendy Auslander, "Child Maltreatment and Bullying Victimization among a Community-based Sample of Sexual Minority Youth: The Meditating Role of Psychological Distress", Journal of Child and Adolescent Trauma, núm. 9 (2016): 283-293. Sarah Dermody, Michael Marshal, Chad Burton Deena y Chisolm, "Risk of Heavy Drinking among Sexual Minority Adolescents: Indirect Pathways through Sexual Orientation-related Victimization and Affiliation with Substance-using Peers". Addiction, núm. 111 (2016): 1599-1606;"1 Gadi Zerach, "Pathological Narcissism, Cyberbullying Victimization and Offending among Homosexual and Heterosexual Participants in Online Dating Websites", Computers in Human Behavior, núm. 57 (2016): 292-299;"41 Ann Desmet, Maddalena Rodelli, Michael Walrave, Gwendolyn Portzky, Eva Dumon y Bart Soenens, "The Moderating Role of Parenting Dimensions in the Association between Traditional or Cyberbullying Victimization and Mental Health among Adolescents of Different Sexual Orientation", International Journal of Environmental Research and Public Health, núm. 18 (2021): 1-20,41 Anna Desmet, Maddalena Rodelli, Michael Walrave, Bart Soenens, Greet Cardon y Ilse De Bourdeaudhuij, "Cyberbullying and Traditional Bullying Involvement among Heterosexual and Non-heterosexual Adolescents, and their Associations with Age and Gender", Computers in Human Behavior, núm. 83 (2018): 254-261. https://doi.org/10.1016/j.chb.2018.02.010, ${ }^{41}$ Antonio Tintori, Giulia Ciancimino, Giorgio Giovanelli y Loredana Cerbara, "Bullying and Cyberbullying among Italian Adolescents: The Influence of Psychosocial Factors on Violent Behaviours", International Journal of Environmental Research and Public Health, núm. 18 (2021): 1-11;41 Kelly Donahue, Niklas Långström, Sebastian Lundström, Paul Lichtenstein y Mats Forsman, "Familial Factors, Victimization, and Psychological Health among Sexual Minority Adolescents in Sweden". American Journal of Public Health, núm. 107 (2017): 322-328.

${ }^{42}$ Elipe, de la Oliva y Del Rey, "Homophobic bullyng and cyberbullyng", 682.

${ }^{43}$ Wang, Chen, Ko, Chang, Lin y Yen, "Effects of Traditional and Cyberhomophobic Bullying", 1312.

${ }^{44}$ Camodeca, Baiocco y Posa, "Homophobic Bullying and Victimization", 516; Desmet, Rodelli, Walrave, Soenens, Cardon y De Bourdeaudhuij, "Cyberbullying and Traditional", 253.

${ }^{45}$ Garaigordobil y Larrain, ““'Bullying and cyberbullying”, 80. 
ansiedad social y síntomas psicopatológicos como somatización y sensibilidad interpersonal. ${ }^{46}$ Del mismo modo, los agresores destacan por una frialdad emocional y desconexión moral. ${ }^{47}$ Sin embargo, no todas las variables son propias de la personalidad, ya que también encontramos que el concepto de identidad social de Tajel ${ }^{48}$ media en este proceso. Los agresores se posicionan en un endogrupo donde tratan de determinar su visión de la realidad como única y hegemónica. En este proceso, los prejuicios, estereotipos y la discriminación afloran, de forma que ejecutan acciones homófobas a la vez que toleran altos niveles de violencia y mantienen altos niveles de autoestima. ${ }^{49}$

Del mismo modo, existen una serie de elementos sociales y ambientales que reducen la probabilidad de ciberacoso. Entre ellos, destacan un consumo bajo de alcohol, una red familiar y de amistad sólida, apoyo social y apoyo institucional. ${ }^{50}$ De forma más concreta, es necesario indicar que el apoyo de la familia reduce la tasa de ansiedad, ${ }^{51}$ a la vez que el control psicológico de las familias se asocia con una mayor idealización suicida. ${ }^{52}$ Del mismo modo, el apoyo institucional y su activismo reduce la prevalencia del ciberacoso hacia los colectivos minoritarios. ${ }^{53}$

Como contribuciones prácticas y futuras líneas de investigación se concluye la necesidad imperiosa de realizar intervenciones de sensibilización y concienciación en los centros de educación secundaria. Aunque las actividades informativas cumplen una importante función y muestran a la sociedad el papel de apoyo de las instituciones, es necesario realizar intervenciones en inteligencia emocional ${ }^{54,55}$. En este sentido, encontramos cómo la frialdad emocional y la desconexión emocional son elementos clave

${ }^{46}$ Ibid., 81.

${ }^{47}$ Camodeca, Baiocco y Posa, "Homophobic Bullying and Victimization", 517.

${ }^{48}$ Henri Tajfel, "Cognitive Aspects of Prejudice", Journal of Social Issues, núm. 25 (1969): 79-97; Henri Tajfel. Introduction à la psychologie sociale (París: Larousse, 1972).

49 Camodeca, Baiocco y Posa, "Homophobic Bullying and Victimization", 512; Garaigordobil y Larrain, “"Bullying and Cyberbullying", 79.

${ }^{50}$ Ibidem.

${ }^{51}$ Wang, Chen, Ko, Chang, Lin y Yen, "Effects of traditional and cyber homophobic bullying", 1311.

${ }^{52}$ Desmet, Rodelli, Walrave, Portzky, Dumon y Soenens, "Risk of heavy drinking”, 3; Donahue, Långström, Lundström, Lichtenstein y Forsman, "Familial factors", 325.

${ }^{53}$ Blaya y Audrin, "Toward an Understanding", 16.

${ }^{54}$ Sofía Buelga, Begoña Iranzo, Maria Jesús Cava y Eva Torralba, "Psychological profile of adolescent cyberbullying aggressors / Perfil psicosocial de adolescentes agresores de cyberbullying", Revista de Psicología Social, núm. 30 (2015): 382-406. https://doi.org/10.1080/21711976.2015.1016754.

55 Fabio Sticca y Sonja Perren, "Is Cyberbullying Worse than Traditional Bullying? Examining the Differential Roles of Medium, Publicity, and Anonymity for the Perceived Severity of Bullying", Journal of Youth and Adolescence, núm. 42 (2013): 739-750. 
en el mantenimiento del ciberacoso. Las intervenciones que parten del desarrollo de competencias emocionales, en las que la empatía y el perdón son las principales claves de acción, refieren mejoras significativas en la reducción del ciberacoso. ${ }^{56}$

Se concluye, entonces, que los estereotipos, los prejuicios y el miedo a la diversidad sexual son factores que contribuyen al mantenimiento del ciberacoso contra el colectivo LGBTIQ+, a la vez que el apoyo social, familiar e institucional se erigen como elementos protectores.

${ }^{56}$ Buelga, Iranzo, Cava y Torralba, "Psychological Profile of Adolescent Cyberbullying Aggressors", 399; Sticca y Perren, "Is Cyberbullying Worse than Traditional Bullying?", 745. 


\section{Bibliografía}

Abreu, Roberto, y Maureen Kenny. "Cyberbullying and LGBTQ Youth: A Systematic Literature Review and Recommendations for Prevention and Intervention". Journal of Child and Adolescent Trauma, núm. 11 (2018): 81-97. https://doi.org/10.1007/s40653-017-0175-7.

Agustín, Santiago. Diversidad sexual en las aulas. Evitar el bullying o acoso. Badajoz: Fundación Trián, 2009.

Benítez, Eduardo (coord.). Ciberbullying LGBT-fóbico. Nuevas formas de intolerancia. Madrid: Grupo de Educación de COGAM, 2016.

Blaya, Catherine, y Catherine Audrin. "Toward an Understanding of the Characteristics of Secondary School Cyberhate Perpetrators". Frontiers in Education, núm. 4 (junio, 2019): 13-19. https://doi.org/10.3389/feduc.2019.00046

Botella, Juan, y Sánchez, Julio. Meta-análisis en ciencias sociales y de la salud. Madrid: Síntesis, 2015.

Bouris, Alida, Bethany Everett, Ryan Heath, Caitlin Elsaesser, y Torsten Neilands. "Effects of Victimization and Violence on Suicidal Ideation and Behaviors among Sexual Minority and Heterosexual Adolescents”. LGBT Health, núm. 3 (2016): 153-161. https://doi.org/10.1089/lgbt.2015.0037.

Buelga, Sofia, Begoña Iranzo, María Jesús Cava y Eva Torralba. "Psychological profile of adolescent cyberbullying aggressors / Perfil psicosocial de adolescentes agresores de cyberbullying”. Revista de Psicología Social, núm. 30 (2015): 382-406. https://doi.org/10.1080/21711976.2015.1016754.

Burton, Chad, Michael Marshal, Chisolm, Deena, Sucato, Gina y Friedman Mark. "Sexual minority-related victimization as a mediator of mental health disparities in sexual minority youth: A longitudinal analysis”. Journal of Youth and Adolescence, núm. 42 (2013): 394-402.

Camodeca, Marina, Roberto Baiocco y Ortensia Posa. "Homophobic bullying and victimization among adolescents: The role of prejudice, moral disengagement, and sexual orientation”. European Journal of Developmental Psychology, núm. 16 (2019): 503-521. https://doi.org/10.1080/17405629.2018.1466699. 
Cifuentes, Francisca, Cristian Carrer y Javier Pascual. “Acoso escolar por orientación sexual, identidad y expresión de género en institutos de educación secundaria catalanes". Revista Nacional e Internacional de Educación Inclusiva, núm. 13 (2020): 153-174.

Collier, Kate, van Beusekom Gabriël, Bos Henny y Sandfort Theo. "Sexual Orientation and Gender Identity/Expression Related Peer Victimization in Adolescence: A Systematic Review of Associated Psychosocial and Health Outcomes". Journal of Sex Research, núm. 50 (2013): 299-317.

Cook, Clayton, Kirk Williams, Nancy Guerra, Tia Kim y Shelly Sadek. "Predictors of Bullying and Victimization in Childhood and Adolescence: A Meta-Analytic Investigation”. School Psychology Quarterly, núm. 25 (2010): 65-83.

Del Olmo, Margarita. "Prejuicios y estereotipos: un replanteamiento de su uso y utilidad como mecanismos sociales”. Revista de Educación XXI, núm. 7 (2005): 13-24.

Dermody, Sarah, Michael Marshal, Chad Burton y Deena Chisolm. "Risk of Heavy Drinking among Sexual Minority Adolescents: Indirect Pathways through Sexual Orientation-Related Victimization and Affiliation with Substance-using Peers". Addiction, núm. 111 (2016): 1599-1606.

Desmet, Ann, Maddalena Rodelli, Michael Walrave, Gwendolyn Portzky, Eva Dumon y Bart Soenens. "The Moderating Role of Parenting Dimensions in the Association between Traditional or Cyberbullying Victimization and Mental Health among Adolescents of Different Sexual Orientation". International Journal of Environmental Research and Public Health, núm. 18 (2021): 1-20.

Desmet, Anna, Maddalena Rodelli, Michael Walrave, Bart Soenens, Greet Cardon y Ilse de Bourdeaudhuij. "Cyberbullying and Traditional Bullying Involvement among Heterosexual and Non-Heterosexual Adolescents, and their Associations with Age and Gender". Computers in Human Behavior, núm. 83 (2018): 254-261. https://doi.org/10.1016/j.chb.2018.02.010.

Donahue, Kelly, Niklas Långström, Sebastian Lundström, Paul Lichtenstein y Mats Forsman. "Familial Factors, Victimization, and Psychological Health Among Sexual Minority Adolescents in Sweden”. American Journal of Public Health, núm. 107 (2017): 322-328. 
Dunn, Hailee, Melissa Clark y Deborah Pearlman. "The Relationship Between Sexual History, Bullying Victimization, and Poor Mental Health Outcomes Among Heterosexual and Sexual Minority High School Students: A Feminist Perspective”. Journal of Interpersonal Violence, núm. 32 (2017): 3497-3519.

Edwards, Katie, Kateryna Sylaska, Johanna Barry, Mary Moynihan, Victoria Banyard, Ellen Cohn, Wendy Walsh y Sally Ward. "Physical Dating Violence, Sexual Violence, and Unwanted Pursuit Victimization: A Comparison of Incidence Rates among Sexual-Minority and Heterosexual College Students". Journal of Interpersonal Violence, núm. $30 \quad$ (2015): 580-600. https://doi.org/10.1177/0886260514535260.

Elipe, Paz, María de la Oliva Muñoz y Rosario del Rey. "Homophobic Bullying and Cyberbullying: Study of a Silenced Problem”. Journal of Homosexuality, núm. 65 (2018): 672-686. https://doi.org/10.1080/00918369.2017.1333809.

Enguix, Begonya. "Activismo y prácticas digitales en la construcción de una esfera LGBTIQ+ en España”. Dados, núm. 59 (2016): 755-787. https://doi.org/10.1590/00115258201691.

Espelage, Dorothy, Kathleen Basile y Merle Hamburger. "Bullying Perpetration and Subsequent Sexual Violence Perpetration among Middle School Students”. Journal of Adolescent Health, núm. 50 (2012): 60-65.

Frost, David, y Ilan Meyer. "Internalized Homophobia and Relationship Quality Among Lesbians, Gay Men, and Bisexuals”. Journal of Counseling Psychology, núm. 56 (2009): 97-109. doi:10.1037/a0012844.

Garaigordobil, Maite, y Enara Larrain. "Bullying and Cyberbullying in LGBT Adolescents: Prevalence and Effects on Mental Health”. Comunicar, núm. 28 (2020): 77-87. https://doi.org/10.3916/C62-2020-07.

Garrido, Rocío, y Zaira Morales. "Una aproximación a la homofobia desde la Psicología”. Propuestas de Intervención Psicología, Conocimiento y Sociedad, núm. 4 (2014): 90-115.

Gegenfurtner, Andreas, y Markus Gebhardt. "Sexuality Education Including Lesbian, Gay, Bisexual, and Transgender (LGBT) Issues in Schools". Educational Research 
Review, núm. $22 \quad$ (septiembre, 2017): 215-222. https://doi.org/10.1016/j.edurev.2017.10.002.

Guasp, April. The School Report. The Experience of Gay Young People in Britain's Schools in 2012. University of Cambridge: Centre for Family Research, 2012.

Hatzenbuehler, Mark, Dustin Duncan y Renee Johnson. "Neighborhood-level LGBT Hate Crimes and Bullying among Sexual Minority Youths: A Geospatial Analysis”. Violence and Victims, núm. 30 (2015): 663-675. https://doi.org/10.1891/08866708.VV-D-13-00166.

Higgins, Julian, y Sally Green. Cochrane Handbook for Systematic Reviews of Interventions Version 5.1.0. London: The Cochrane Collaboration, 2011.

Hinduja, Sameer, y Justin Patchin. Bullying, Cyberbullying and Sexual Orientation. Florida: Cyberbullying Research Center, 2011.

Kosciw, Joseph, Emily Greytak, Noreen Giga, Christian Villenas y David Danischewski. The 2015 National School Climate Survey: The Experiences Of Lesbian, Gay, Bisexual, Transgender, And Queer Youth In Our Nation's Schools. New York: GLSEN, 2016.

Larrain, Enara, y Maite Garaigordobil. "El bullying en el País Vasco: Prevalencia y diferencias en función del sexo y la orientación-Sexual”. Clínica y Salud, núm. 31 (2020): 147-153.

Martxueta, Aitor, y Juan Etxeberria. "Claves para atender la diversidad afectivo-sexual en el contexto educativo desde un enfoque global escolar". Revista Española de Orientación y Psicopedagogía, núm. 25 (2014): 121-128.

Mayock, Paula, Audrey Bryan, Nicola Carr y Karl Kitching. Supporting LGBT Lives: A Study of the Mental Health and Well-being of Lesbian. Gay, Bisexual and Transgender People. Dublin: National office of suicide prevention, GLEN and belong to youth service, 2009.

Mamédio da Costa Santos, Cristina, Cibele de Mattos Pimenta y Moacyr Cuce Nobre. "Estrategia pico para la construcción de la pregunta de investigación y la búsqueda de evidencias". Revista Latino-Americana de Enfermagem, núm. 15, (2007): 2-4. 
Meyer, Ilan. "Prejudice, Social Stress, and Mental Health in Lesbian, Gay, and Bisexual Populations: Conceptual Issues and Research Evidence". Psychological Bulletin, núm. 129 (2003): 674-697.

Moher, David, Alessandro Liberati, Jennifer Tetzlaff, Douglas Altman y PRISMA, "Preferred Reporting Items for Systematic Reviews and MetaAnalyses: The PRISMA Statement". PLoS Medicine, núm. 6 (2009): e1000097. https://doi.org/10.1371/journal.pmed.1000097.

Moreau, David, y Beau Gamble. Conducting a Meta-analysis in the Age of Open Science: Tools, Tips, and Practical Recommendations. Auckland: The University of Auckland, 2020.

Otis, Melanie, Carrie Oser y Staton Michele -Tindall. "Violent Victimization and Substance Dependency: Comparing Rural Incarcerated Heterosexual and Sexual Minority Women". Journal of Social Work Practice in the Addictions, núm. 16 (2016): 176-201.

Parales-Quenza, Carlos, y Milcíades Vizcaíno-Gutiérrez. "Las relaciones entre actitudes y representaciones sociales: elementos para una integración conceptual". Revista Latinoamericana de Psicología, núm. 39 (2007): 351-361.

Pichardo, José Ignacio. Abrazar la diversidad: propuestas para una educación libre de acoso homofóbico y transfóbico. Madrid: Instituto de la mujer y para la igualdad de oportunidades, 2015.

Ponzetti, James. Evidence-based Approaches to Sexuality Education. A Global Perspective. New York: Routledge, 2016.

Rankin, Susan, Genevieve Weber, Warren Blumenfeld y Frazer Somjen. State of Higher Education for Lesbian, Gay, Bisexual, and Transgender People: 2010 National College Climate Survey. Charlotte, NC: Campus Pride, 2010.

Ramsey, Jaimi, Lisabeth DiLalla y Megan McCrary. "Cyber Victimization and Depressive Symptoms in Sexual Minority College Students". Journal of School Violence, núm. 15 (2016): 483-502.

Ruiz, Carmen. "Estrategias para educar en y para la igualdad: coeducar en los centros". ATLÁNTICAS - Revista Internacional de Estudios Feministas, núm. 2 (2017): 166191. 
Socarrás, Delia, Massiel Nuñez, Martín del Río Marais, Ada Rodríguez y Gisett Gutiérrez. "Estudio exploratorio retrospectivo sobre violencia homofóbica y transfóbica en la trayectoria escolar de activistas cubanos LGBT". Revista Sexología y Sociedad, núm. 25 (2019): 72-87.

Sterzing, Paul, Jun Sung Hong, Rachel Gartner y Wendy Auslander. "Child Maltreatment and Bullying Victimization among a Community-based Sample of Sexual Minority Youth: The Meditating role of Psychological Distress". Journal of Child and Adolescent Trauma, núm. 9 (2016): 283-293.

Sticca, Fabio, y Sonja Perren. "Is Cyberbullying Worse than Traditional Bullying? Examining the Differential Roles of Medium, Publicity, and Anonymity for the Perceived Severity of Bullying”. Journal of Youth and Adolescence, núm. 42 (2013): 739-750.

Tait, Robert. “Alcohol-related Victimisation: Differences between Sexual Minorities and Heterosexuals in an Australian National Sample”. Drug and Alcohol Review, núm. 34 (2015): 366-374.

Tajfel, Henri. “Cognitive Aspects of Prejudice”. Journal of Social Issues, núm. 25 (1969): 79-97.

Tajfel, Henri. Introduction à la psychologie sociale. París: Larousse, 1972

Tintori, Antonio, Giulia Ciancimino, Giorgio Giovanelli y Loredana Cerbara. "Bullying and Cyberbullying among Italian Adolescents: The Influence of Psychosocial Factors on Violent Behaviours". International Journal of Environmental Research and Public Health, núm. 18 (2021): 1-11.

Vizcaíno Verdú, Arantxa, Paloma Contreras Pulido y María Dolores Guzmán Franco. “'La Bella y la Bestia' y el movimiento LGBTIQ+ en Disney: empoderamiento y activismo en YouTube”. Área Abierta, núm. 20, (2020): 75-93.

Wang, Lihien-Chuan, Mu-Hong Chen, Nai-Ying Ko, Yu-Ping Chang, Lin Huang-Chi y Cheng-Fan Yen. "Effects of Traditional and Cyber Homophobic Bullying in Childhood on Depression, Anxiety, and Physical Pain in Emerging Adulthood and the Moderating Effects of Social Support among Gay and Bisexual Men in Taiwan”. Neuropsychiatric Disease and Treatment, núm. 14 (2018): 1309-1317. 
Zerach, Gadi. "Pathological Narcissism, Cyberbullying Victimization and Offending among Homosexual and Heterosexual Participants in online Dating Websites". Computers in Human Behavior, núm. 57 (2016): 292-99.

Young, Iris M. “La democracia y 'el otro': más allá de la democracia deliberativa”, trad. de Lelia Mooney Sirotinsky. Revista Jurídica de la Universidad de Palermo, Año 5, núm. 1 (agosto, 2000): 41-56. http://www.palermo.edu/derecho/publicaciones/pdfs/revista_juridica/n5N12000/051 Juridica03.pdf.

Young, Iris Marion. La justicia y la política de la diferencia. Madrid: Cátedra, 2000.

Young, Iris Marion. Responsabilidad por la justicia. Madrid: Morata, 2011. 